\title{
A Documentation Generator for (C)LP Systems
}

\author{
Manuel Hermenegildo \\ herme@fi.upm.es \\ Department of Computer Science, Technical U. of Madrid (UPM)
}

\begin{abstract}
We describe lpdoc, a tool which generates documentation manuals automatically from one or more logic program source files, written in Ciao, ISO-Prolog, and other (C)LP languages. It is particularly useful for documenting library modules, for which it automatically generates a rich description of the module interface. However, it can also be used quite successfully to document full applications. A fundamental advantage of using 1pdoc is that it helps maintaining a true correspondence between the program and its documentation, and also identifying precisely to what version of the program a given printed manual corresponds. The quality of the documentation generated can be greatly enhanced by including within the program text assertions (declarations with types, modes, etc. ...) for the predicates in the program, and machine-readable comments. One of the main novelties of 1 pdoc is that these assertions and comments are written using the Ciao system assertion language, which is also the language of communication between the compiler and the user and between the components of the compiler. This allows a significant synergy among specification, debugging, documentation, optimization, etc. A simple compatibility library allows conventional (C)LP systems to ignore these assertions and comments and treat normally programs documented in this way. The documentation can be generated interactively from emacs or from the command line, in many formats including texinfo, dvi, ps, pdf, info, ascii, html/css, Unix nroff/man, Windows help, etc., and can include bibliographic citations and images. lpdoc can also generate "man" pages (Unix man page format), nicely formatted plain ASCII "readme" files, installation scripts useful when the manuals are included in software distributions, brief descriptions in html/css or info formats suitable for inclusion in on-line indices of manuals, and even complete WWW and info sites containing on-line catalogs of documents and software distributions. The lpdoc manual, all other Ciao system manuals, and parts of this paper are generated by lpdoc.
\end{abstract}

\section{Introduction}

lpdoc is an automatic program documentation generator for (C)LP systems. Its main functionality is to generate a reference manual automatically from one or more source files of (constraint) logic programming systems. It has been developed as part of the Ciao Prolog [1] program development environment, but it 


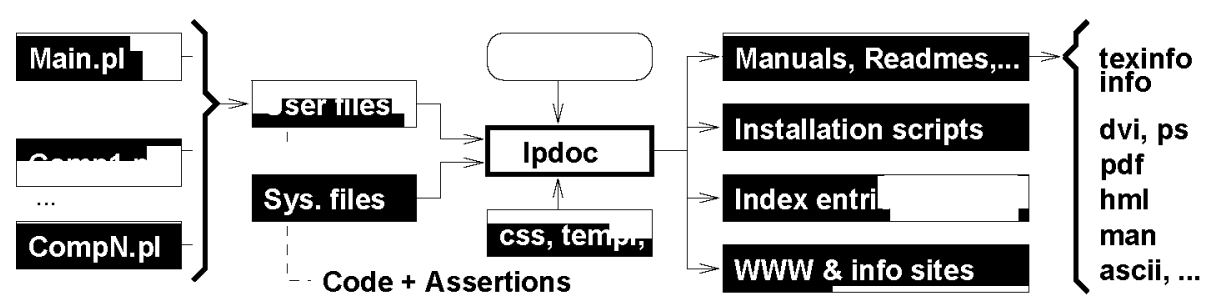

Fig. 1. Overall operation

can also be used to document source files of almost any other (ISO-)Prolog-like [6] (C)LP system. Ipdoc is particularly useful for documenting library modules, for which it automatically generates a rich description of the module interface. However, it can also be used quite successfully to document full applications.

The operation of Ipdoc is illustrated in Figure 1. Ipdoc combines the information from a number of user and system files (as specified in a user-provided configuration file-SETTINGS in Figure 1$)^{1}$ and produces manuals in a number of formats (texinfo, dvi, ps, pdf, info, html/css, ascii, Windows help, etc.) which can include bibliographic citations and images (if the target supports them). In addition to full manuals, lpdoc can also generate nicely formatted plain ASCII "readme" files, man pages (Unix manual page format), as well as brief descriptions in html or emacs info formats suitable for inclusion in an online master index of applications. Using these index entries, lpdoc can create and maintain fully automatically WWW and info sites containing pointers to the on-line versions of the documents it produces. Similarly, it can be used to generate software distribution sites. 1pdoc also generates installation scripts for the manuals it produces, which simplify the process of creating a distribution of the corresponding software package. Finally, it is also possible to start a number of viewers directly from lpdoc in order to quickly browse the manuals produced. The documentation can be generated interactively from emacs or from the command line in a documentation directory containing configuration files.

The quality of the documentation generated can be greatly enhanced by including within the program text assertions (declarations with types, modes, and other properties) for the predicates in the program, and machine-readable comments (in the "literate programming" style [11,4]). The assertions and comments included in the source file need to be written using the Ciao assertion language $[12,10,13]$. This is one of the main novelties of 1 pdoc. The fact that this assertion language also serves as the communication vehicle between the compiler and the user and between the components of the compiler allows a significant synergy among specification, debugging, analysis, optimization, and, thanks to lpdoc, program documentation. As we will see, Ipdoc understands natively this language and can thus provide accurate information and relate both the the formal and the textual aspects of properties with the assertions in which they occur.

\footnotetext{
${ }^{1}$ It also possible to use files written in GNU texinfo format as part of the lpdoc input (useful when gradually converting a manual from this popular format to lpdoc).
} 
In order to make the discussion self-contained, an example of source code and the output produced by lpdoc is included at the end of the paper. However, since it is difficult to show significant output from the system in the space available, the reader is invited to look at actual manuals generated by lpdoc for reference while reading the paper. In particular, the lpdoc manual [9] and all other Ciao system manuals are generated by lpdoc. The Ciao manuals and other lpdoc-generated manuals can be found on-line at http://www.clip.dia.fi.upm.es/Software, http://www.clip.dia.fi.upm.es/Software/Ciao, and http://www.clip.dia.fi.upm.es/Software/Beta (registration as a Beta tester is needed for access to the latter). In fact, all these WWW sites are automatically generated and maintained by lpdoc as well.

\section{Generating a manual}

We now describe, from the user's point of view, the process of generating a manual (semi-)automatically from a set of source files, installing them in a public area, and accessing them on line.

Documentation can be generated fully automatically from within emacs (e.g., from the Ciao emacs-based program development environment) or calling lpdoc from the command line. The process starts (automatically in the former case or by hand in the latter) by creating a directory (e.g., doc) in which the documentation will be built. This directory is usually placed in the top directory of the distribution of the application or library to be documented and will contain the (automatically generated) manuals as well as scripts for installation of such manuals during the installation of the software package. Typically, almost all files in this directory will be automatically generated by lpdoc, which also takes care of cleaning up this directory of intermediate files before distribution of the software, leaving only the manuals in the selected formats. The configuration file of Figure 1, normally named SETTINGS, also resides in this directory. This file is written in Prolog syntax, possibly using Ciao syntactic enhancements (in particular, the functional notation is often useful in this context).

A manual can be generated either from a single source file or from a set of source files. In the latter case, one of these files should be chosen to be the main file, and the others will be the component files. The main file is the one that will provide the title, author, date, summary, etc. to the entire document. In principle, any set of source files can be documented, even if they contain no assertions or comments. However, the presence of these will greatly improve the documentation (see Section 3).

The name of main file is specified in the SETTINGS file by defining a fact of a predicate main. Facts of a (possibly empty) predicate components define the component files which will generate the different chapters of the manual. Facts of a predicate filepaths are used to define all the directories where the previously mentioned files can be found. Similarly, facts of the predicate systempaths are used to list all the system directories where system files used by the files being documented can be found. This is needed because on startup lpdoc has no 
default search paths for files defined, not even those defined by default in the Prolog system under which it was compiled (typically Ciao). This has the important consequence that it allows documenting Prolog systems other than that under which Ipdoc was compiled. The effect of putting a path in systempaths instead of in filepaths is that the modules and files in those paths are documented as system modules (this is useful when documenting an application to distinguish its parts from those which are in the system libraries).

These are the only settings which are strictly needed in order to generate a manual. However, many aspects of the generated manuals can be controlled through additional configuration parameters. For example, it is possible to control what is included in the different files and how: whether to include bug information or not, comments associated to version changes and/or to patches, author info, detailed explanation of predicate argument modes, starting page number, etc. It is also possible to define the set of formats (dvi, ps, pdf, ascii, html, info, manl, ...) in which the documentation should be generated by default (however, a manual in any of the supported formats can be generated on demand by typing "lpdoc format"). In particular, selecting htmlindex and/or infoindex requests the generation of (parts of) a master index to be placed in an installation directory and which provide pointers to the documents generated.

A predicate indices determines a list of indices to be included at the end of the document. These can include indices for defined predicates, modules, properties, types, concepts, files, etc. The contents of these indices are afterwards used for several purposes in on-line documents. In particular, lpdoc includes an emacs library for automatically locating any part of the manual related to the symbol (predicate, flag, property, type, etc.) under the cursor ("help for symbol under cursor") and also performing automatic completion of partially typed names of predicates, types, etc. This is very useful when typing the name of a library predicate: it is possible to complete the name and also locate in one step the corresponding page in the on-line manual generated by lpdoc.

It is possible to define a predicate bibfile containing paths of .bib files, i.e., files containing bibliographic entries in $\mathrm{BiBTeX}$ format. If citations are used in the text (using the @cite command) these will be the files in which the citations will be searched for. All the references in all component files will appear together in a References appendix at the end of the manual (the -norefs option prevents generation of the 'References' appendix). It is also possible to select different levels of verbosity during processing, from pretty silent -more or less only a couple of messages per file-, to quite verbose, reporting the files visited and the predicates being documented on the fly. The latter is obviously quite useful for debugging.

Once the manual has been generated in the desired formats, lpdoc can also install them in a different area, specified by a predicate docdir in the SETTINGS file. As mentioned before, lpdoc can generate directly brief descriptions in html or emacs info formats suitable for inclusion in an on-line index of applications. In particular, if the htmlindex and/or infoindex options are selected, then lpdoc will create the installation directory, place the documentation in the de- 
sired formats in this directory, and produce and place in the same directory suitable index.html and/or dir files. These files will contain some basic info on the manual (extracted from the summary and title, respectively) and include pointers to the relevant documents which have been installed. The appearance of the actual indices created (e.g., index.html) can be controlled via templates and style sheets, specified in the configuration file. Several manuals, coming from different doc directories, can be installed in the same docdir directory. In this case, the descriptions of and pointers to the different manuals will be automatically combined (appearing in alphabetic order) in the index.html and/or dir indices, and a contents area will appear at the beginning of the html index page. In the same way, facilities are provided for de-installation of manuals from the docdir area.

\section{Enhancing the documentation being generated}

lpdoc will generate quite useful information from standard program files: e.g., exported predicates with their arity, characteristics of these predicates-dynamic, multifile, ...-, other modules used, required libraries, and, if available, types and other properties, etc. However, the quality of the documentation generated can be greatly enhanced by including within the program text assertions, and machine-readable comments.

Assertions are declarations which are included in the source and provide information regarding certain characteristics of the program. Typical assertions include type declarations, modes, general properties (such as does not fail), etc. For our purposes, we can consider standard compiler directives (such as dynamic/1, op/3, meta_predicate/1...), also as assertions. When documenting a module, lpdoc will use the assertions associated with the module interface to construct a textual description of this interface. In principle, only the exported predicates are documented, although any predicate can be included in the documentation by explicitly requesting it (by using a particular comment/2 declaration -see below). Judicious use of these assertions allows at the same time documenting the program code, documenting the external use of the module, and greatly improving the debugging process. The latter is possible because the assertions provide the compiler with information on the intended meaning or behavior of the program (i.e., the specification) which can be checked at compile-time (by a preprocessor/static analyzer) and/or at run-time (via checks inserted by the same preprocessor) -see [8] for details.

Machine-readable comments are also declarations included in the source program but which contain additional information intended to be read by humans (this is where the connection with the literate programming style of Knuth [11, $4]$ is closest). These declarations are ignored by the compiler in the same way as classical comments. Thus, they can be used to document the program source in place of (or in combination with) the normal comments typically inserted in the code by programmers. However, because they are more structured and they are machine-readable, they can also be used to improve the automatic generation of 
printed or on-line documentation. Typical such comments include module title, author(s), bugs, changelog, etc. Judicious use of these comments allows enhancing at the same time the documentation of the program text and the manuals generated.

As mentioned before, Ipdoc requires these assertions and comments to be written using the Ciao system assertion language $[12,10,13] .^{2}$ Comments have the general form:

:- comment (Comment Type, CommentData)..$^{3}$

where generally the first argument states the type of comment and the second one the comment itself, written in a particular markup language which is very similar to texinfo and LaTeX (see Section 7). Examples of comments are:

:- comment(title, "Complex numbers library").

:- comment(summary, "Provides an ADT for complex numbers.").

:- comment (ctimes $(\mathrm{X}, \mathrm{Y}, \mathrm{Z})$, "@var $\{\mathrm{Z}\}$ is @var $\{\mathrm{Y}\}$ times @var $\{\mathrm{X}\} . ")$.

An example of an assertion is:

$:-\operatorname{pred}$ qsort $(X, Y): \operatorname{list}(X) \Rightarrow \operatorname{sorted}(Y)$

\# "@var $\{Y\}$ is a sorted permutation of @var $\{\mathrm{X}\} . "$.

which states that in the calls to predicate qsort/2 the first argument should be a list and, upon exit, the second argument should be sorted. There is also a textual assertion comment, written using the same markup language as in comment/2. The properties list/1 and sorted/1 used in the assertion might be declared as such with the following assertions (we are also including the actual definitions for illustration purposes):

:- prop sorted(X) \# "@var $\{\mathrm{X}\}$ is sorted.".

sorted ([]).

$\operatorname{sorted}\left(\left[{ }_{-}\right]\right)$.

$\operatorname{sorted}([X, Y \mid R]):-X<Y, \operatorname{sorted}([Y \mid R])$.

:- regtype list $(\mathrm{X})$ \# "@var $\{\mathrm{X}\}$ is a list.".

list ([]).

list $\left(\left[{ }_{-} \mid \mathrm{T}\right]\right)$ : - list $(\mathrm{T})$.

(list is actually a particular case of property: a regular type). Space limitations unfortunately do not allow a description of the assertion language. See the appendices for more examples and $[12,10,13]$ for details.

\section{Overall structure of the generated documents}

If the manual is generated from a single main file (i.e., components is empty), then the document generated will be a flat document containing no chapters. If the manual is generated from a main file and one or more components, then the

\footnotetext{
${ }^{2}$ A simple compatibility library can be used so that programs documented using assertions and comments can be loaded by traditional (constraint) logic programming systems which lack native support for them. Using this library, such assertions and comments are simply ignored by the compiler.

${ }^{3}$ For brevity, also :- $\operatorname{doc}(\ldots, \ldots)$. can be used.
} 
main file will be used to generate the cover and introduction, while each of the component files will generate a separate chapter. The contents of each chapter will reflect the contents of the corresponding component source file.

If a .pl file does not define the predicates main/ 0 or main $/ 1$, it is assumed to be a library and information on the interface (e.g., the predicates exported by the file, the name of the module and usage if it is a module, etc. -the API), is produced by default. If, on the contrary, the file defines the predicates main $/ 0$ or main $/ 1$, it is assumed to be an application and no description of the interface is generated. Instead, usage information is produced. Any combination of libraries and/or main files of applications can be used arbitrarily as components or main files of an lpdoc manual (see the lpdoc manual [9] for interesting combinations). A :-comment (filetype, filetype). declaration can be used to defeat these rules.

In any case, a cover is generated with the title, authors, summary, version, etc. of the whole manual, which are those of the main file. Then comes the table of contents, whose level of detail can also be controlled via options. This is followed by the sections or chapters corresponding to the file or files being documented. Finally, the manual ends with the selected indices, list of references, etc.

\section{$5 \quad$ Structure of chapters}

The structure of the individual chapters depends also on whether they are applications or libraries. In the case of libraries, the structure is as below. Note that inclusion of many of the following items can be turned on or off and can be configured in several ways through options. Examples of a source file and the chapter generated for it (under a particular set of options) are listed in appendices $\mathrm{A}$ and $\mathrm{B}$, for illustration while reading the following items.

- Chapter title, from a title comment, such as the line:

:- comment(title,"The classical quick-sort").

in the example. If the file is the main file, the title text (a documentation string) will also be used in the cover page and also as the description of the manual in on-line indices. If no such comment exists, then a suitable one is generated from the module or file name. Also, a subtitle comment is allowed.

- Authors, which are obtained from author comments, such as: :- comment (author, "Alan Robinson").

There can be more than one of these declarations per module (normally, one per author). These are followed by copyright info (from copyright comments) and version info (from changelog comments). If the file is part of a bigger package, then both the file version (i.e., when last changed) and the overall system versions are documented.

- Chapter introduction, taken from a summary comment or from a module comment, if no summary is available (see also the example).

- A usage and interface section, which is typically generated without any need for comment declarations, and includes: 
- Module usage info, stating whether it is a module, a user file, a package [2], etc., and how it is to be loaded. These automatically generated loading instructions can be replaced by more specific ones by means of a usage comment.

- List of exported predicates. These are classified by kind: normal predicates, multifile predicates, regular types, properties, declarations, etc.

- The list of other modules used. These are separated into User, System and, optionally, Engine libraries ${ }^{4}$ (this division is controlled by the paths in SETTINGS). It is possible to optionally prevent the information on System and/or Engine libraries used from being included in the manual. Note that this information is useful because it allows the user of a library to see which other libraries it will load, and thus the impact that it will have on the size of the executable.

- A section with overall information on the library, taken from the module comment, if available (and if this comment was not already used before).

- A section documenting new declarations [2] defined (Ciao-specific).

- A section documenting the predicates (including regular types and properties) exported by the library (e.g., qsort/2, list/1, and sorted/1 in the example). In principle, all exported predicates are documented. However, it is possible to prevent documentation on a predicate from appearing in the manual by using a hide comment (useful, e.g., for low-level predicates which are exported but are not meant to be used directly).

- A section documenting the multifile predicates defined by the library.

- Possibly a section documenting some internal predicates (or regular types or properties) defined by the library. In principle internal (local) predicates are not documented, but documentation of an internal predicate can be forced by using a doinclude comment. This is the case for partition/4 in the example. This is useful for example when generating "internals" manuals or implementation chapters for inclusion in larger documents.

- Optionally, a section with known bugs, i.e., those present in bug comments (see the example).

- Optionally, a section with a list of changes, those present in version comments (see the example). It is possible to list only comments associated with major version changes an leave out minor changes ("patches"). This allows writing version comments which are internal, i.e., not meant to appear in the manual. Code is provided for maintaining version numbers automatically with emacs, or they can also be maintained with other tools such as standard version control systems.

- Reexported predicates, i.e., predicates which are exported by a module $\mathrm{m} 1$ but defined in another module $\mathrm{m} 2$ which is used by $\mathrm{m} 1$, are normally not documented in the original module, but instead a simple reference is included to the module in which they are defined. This is useful if the documentation for the referred module is included in the same document. Otherwise, using a

\footnotetext{
${ }^{4}$ In Ciao, engine libraries contain builtins that are always present in any executable, independently of whether they are imported or not from the program.
} 
comment/ 2 declaration with doinclude in the first argument and the predicate descriptor in the second forces the documentation to be included in the referring module. This is often useful when documenting a library made of several components: typically there is a principal module, which is the one which users will do a use_module/1 of, and which exports or reexports all the predicates which define the library's user interface. It is then often best to include in the manual this main file only, with the appropriate doincludes.

If the chapter is documenting an application, then no module interface information is included in the documentation, but it still contains title, authors, version, summary, usage information, body, bugs, changelog, etc.

\section{Documentation on predicates, properties, etc.}

We now describe how individual predicates, declarations, properties, etc. are documented. This is done in essentially the same way, independently of whether they appear in the export list or they are internal predicates. The documentation is obviously more detailed if more information is available on the predicate in the form of assertions and comments.

If the program does not contain any declarations for the predicate, a line is output documenting that this is a predicate of the given name and arity and a simple comment is included saying that there is no further documentation available. Note that this means for example that the predicate will appear in the index, and also that its name will be available for command completion within emacs.

If the predicate is declared to be a property or regular type, then this fact is included in the documentation. If there is no textual comment available for it, then its actual definition is included in the documentation (see list/1 in the example). Otherwise, the comment is used (as with sorted $/ 1$ in the example).

If an overall comment (a comment/ 2 declaration) is available for a predicate, it is used as a general explanation (see the general comment for qsort/2 in the example). If any assertions are present, they are documented in mostly textual form. In particular, if pred declarations are present, each of them is considered a possible conceptual usage (i.e., a particular way in which the predicate is intended to be used) and is documented as such (e.g., the two pred declarations for qsort $/ 2$ in the example). Also, if a comment appears in the pred declaration, it is associated with the usage (as opposed to the general comment above).

The syntactic sugar which can be used with the assertions (e.g., property macros $[12,13]$ ) can be either kept as is or expanded when documentation is generated. In the example, having chosen the corresponding option, the modes (which are "property macros" in the Ciao assertion language) used in partition/4 have been spelled out in the documentation. Note that the parametric type list/2 used (e.g., in list ( $\mathrm{X}, \mathrm{num})$ ) is assumed to be imported by default.

A point of particular interest is that if a textual comment is available in the definition of a property or regular type (such as for sorted in the example) then this text is used when the property itself is used elsewhere in an assertion. An 
example is the use of sorted in the two usages for qsort/2. This also occurs if the property is imported from another module: the comment is read from that module (actually, from the module's .asr interface file) $[14] .^{5}$

\section{Documentation strings}

As shown in previous examples, the character strings which can be used in machine readable comments (comment/2 declarations) and assertions can include certain formatting commands ("markup"). The syntax of all the formatting commands is: @command (followed by either a space or \{\} ), or Qcommand $\{$ body $\}$ where command is the command name and body is the (possibly empty) command body. Also, a command may have several bodies, as in: @command $\{$ body 1$\}\{$ body2 $\}$.

In order to make it possible to produce documentation in a wide variety of formats, the command set is kept small. The names of the commands are intended to be reminiscent of the commands used in the LaTeX text formatting system, except that "Q" is used instead of "V". Note that " " would need to be escaped in ISO-Prolog strings, which would make the source less readable. ${ }^{6}$ Given that space restrictions do not allow a full description of the command set, we provide a general description by categories.

There are a number of indexing commands which are used to mark certain words or sentences in the text as concepts, names of predicates, libraries, files, etc. and which then get indexed and cross-referenced in hypertext formats. There are also referencing commands which are used to introduce bibliographic citations and references to sections, urls, email addresses, etc. A set of formatting commands are provided which allow typesetting certain words or sentences in a special fonts/faces, build itemized lists, introduce sections, include verbatim examples, cartouches, etc. There are also special commands for generating accented and special characters. A number of inclusion commands (@include, @includedef,...) allow inserting code or strings of text as part of the documentation. The latter may reside in external files or in the file being documented. The former must be part of the module being documented. There are also commands for inserting and scaling images.

\section{Other issues}

Separating the documentation from the source file: Sometimes one would not like to include long introductory comments in the module itself but would rather have them in a different file. This can be done quite simply by using the oinclude command mentioned above. For example, the following declaration:

:- comment (module, "@include\{Intro.lpdoc\}").

\footnotetext{
${ }^{5}$ This occurs in the example with list/2, which is in the lists library.

${ }^{6} \mathbb{Q}$ is familiar to texinfo users and, in any case, many ideas in LaTeX were taken from scribe, where the escape character was indeed "ब"?
} 


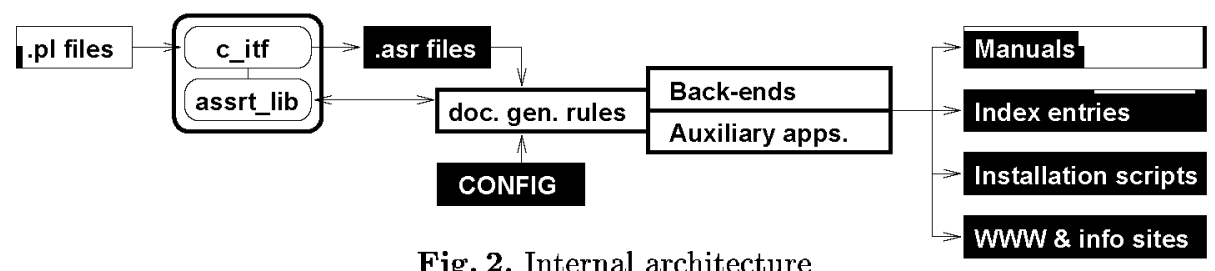

will include the contents of the file Intro.Ipdoc as the module description.

Alternatively, sometimes one may want to generate the documentation from a completely different file. Assuming that the original module is $\mathrm{m} 1 . \mathrm{pl}$, this can be done by calling the module containing the documentation m1_doc.pl. This m1_doc.pl file is the one that will be included the lpdoc SETTINGS file, instead of $\mathrm{m} 1 . \mathrm{pl}$. lpdoc recognizes and treats such _doc files specially so that the name without the _doc part is used in the different parts of the documentation, in the same way as if the documentation were placed in file $\mathrm{m} 1$.

Generating auxiliary files (e.g., READMEs): Using lpdoc it is often possible to use a common source for documentation text which should appear in several places. For example, assume a file INSTALL.lpdoc contains text (with lpdoc formatting commands) describing an application. This text can be included in a section of the main file documentation as follows:

$$
\begin{aligned}
:- \text { comment (module,"... } & \text { @section Installation instructions\} } \\
& \text { Qinclude\{InSTALL.lpdoc\} ..."). }
\end{aligned}
$$

At the same time, this text can be used to generate a nicely formatted INSTALL file in ASCII, which can perhaps be included in the top level of the application's source directory. To this end, an INSTALL.pl file is constructed as follows:

:- include(library([assertions])).

:- comment (title, "Installation instructions").

:- comment (module, "@include\{INSTALL.lpdoc\}").

main. $\%$ forces file to be documented as an aplication

Then, the ASCII INSTALL file will be generated by simply running lpdoc ascii in a directory with a SETTINGS file where MAIN is set to INSTALL.pl (these steps can be performed automatically in the interactive environment).

\section{System architecture and implementation}

Space limitations only allow us to sketch the architecture and implementation of the system. ${ }^{7}$ Ipdoc is implemented in (Ciao-)Prolog and compiled into a standalone Ciao executable. Executable size is around $300 \mathrm{~K}$ for the dynamic version and $2.7 \mathrm{Mbytes}$ for the fully static version (including WAM engine). The

\footnotetext{
${ }^{7}$ Details can be found in the comments within the source files of the system, which, when printed out using lpdoc constitute the system's internals manual.
} 
executable is generated from around $11 \mathrm{~K}$ lines of application-specific code (including comments/documentation) and $12 \mathrm{~K}$ lines from the Ciao system libraries, plus some $1 \mathrm{~K}$ additional lines of miscellaneous code $(\mathrm{html} / \mathrm{css}, \mathrm{TeX}$ and $\mathrm{BiBTeX}$ styles, emacs lisp, etc.). The first version of the system was completed between 1996 and 1997 with successive improved versions appearing after that.

Since the source used by lpdoc is not just simple comments but the actual code of the modules (e.g., the assertions, the module declarations, exports, imports, dynamic declarations, syntax extensions, mode definitions, etc., and even the source code) Ipdoc requires a full reader. This is specially true for the full Ciao system source language, which is designed to be very extensible [2]. Also, the reader (and the overall system) must be adaptable to different operator definitions and sets of built-ins so that different flavors of Prolog and other (C)LP languages can be supported. Finally, because the design objective was to be able to document very large systems in an efficient way, processing of the source files, including module interface information, declarations, comments, assertions, etc. needs to be highly incremental.

At the level of source file processing, the objectives are achieved in a relatively straightforward way thanks to the Ciao assertion processing library (see Figure 2), itself an instance of the c_itf low-level generic modular processing library [3]. For each documented file, and transitively for other files used by the one being documented, the library reads all the information, normalizes the assertions, and saves them in .asr and .itf cache files. This process is only repeated on a needed basis when a source file is modified. The syntax extensions and builtins "seen" during the processing of a file can be controlled by setting the lpdoc load paths (systempaths and filepaths -see Section 2) so that files containing the appropriate syntax extension definitions and the documentation for the builtins are "seen" by lpdoc (see [2] for details).

Once it has read the information for a file and its auxiliary files, lpdoc uses a number of documentation generation rules (also written in Prolog and part of which are defined in a configuration file) to implement the documentation actions outlined in previous sections. Documentation is in general first generated in an internal format (basically, the language of Section 7), and then converted by a number of backends in Prolog and/or auxiliary (publicly available) applications ( $\mathrm{TeX}$, dvi2ps, etc.) into manuals in the different formats, index entries, installation scripts, etc. It is quite easy to add new backends. The generation of the documentation files is also partly incremental, in that a documentation cache file (currently in GNU texinfo format) ${ }^{8}$ is kept for each Prolog file being documented and which only changes as needed by any changes in the source files. Thus, a form of "separate documentation" (in the same sense as "separate compilation") is achieved. Early versions used makefiles for dependency tracking in this process, while more recent versions do the job in Prolog using the ciao make library, which has greatly increased portability. Unfortunately some of the auxiliary tools currently used by lpdoc are difficult to make incremental,

\footnotetext{
8 See "The GNU Texinfo Documentation System" manual for more info on this format, widely used in the GNU project and on Linux and other Unix systems.
} 
although this is not a real problem in practice. For example, the Ciao reference manual is generated from approx. 180 source Prolog files and a corresponding number of cache texinfo files, producing $50 \mathrm{~K}$ lines of texinfo code and 550 busy A4 pages. Regenerating the dvi file after changing a single file (e.g., the lists library) takes only $10 \%$ of the time needed to generate the whole manual from scratch.

One of the most complicated issues has been to generate consistent documentation and support as many common features as possible across many different formats. For example, supporting citations using $\mathrm{BiBTeX}$ files was tricky because few of the underlying formats were capable of this (the solution was to bridge the missing capabilities in Prolog).

\section{Related work}

We are not aware of other automatic documentation systems that have all the capabilities of lpdoc. There are some systems which allow interspersing TeX and Prolog in a source file in the style of Knuth's original formulation of literate programming. ${ }^{9}$ While these systems are quite useful, we believe that 1 pdoc goes beyond them in that a significant part of the documentation is generated essentially automatically by modules of the compiler, and that the assertion language used is shared with other program development tools, which makes them quite useful beyond just documentation. ICON and Perl have some (limited) facilities for merging documentation and programs. Perhaps the closest tool to lpdoc is the javadoc documentation system for Java [7] (the development of lpdoc and javadoc started about the same time and independently). As lpdoc, javadoc uses information which is typically read and/or derived by the compiler (types, class structure, etc.), allows including textual comments with (HTML) markup, and can be extended via doclets. javadoc seems to have concentrated on producing good HTML output, while lpdoc aims to produce consistent documentation across a large number of different formats. Because of the tight integration with the language, javadoc cannot be used well for Prolog programs (in the same way as Ipdoc would certainly not be as effective as javadoc for Java programs). Also, we feel that the markup language and, specially, the assertion language and the way properties can be used in documentation, are richer in lpdoc. Also, lpdoc is not limited to documenting APIs, i.e., it can also include source code in the generated documents, create indices, maintain web and info sites, etc.

\section{Conclusions}

Since the first "production" versions of the lpdoc system became available [9], we have applied it in a number of scenarios. We have used it to document all the components of the Ciao Prolog development environment, libraries for SICStus

\footnotetext{
${ }^{9}$ See $\mathrm{ftp}: / / \mathrm{f} t \mathrm{p}$.dante.de/tex-archive/macros/latex/contrib/other/gene/pl.tar.gz for a good example.
} 
and CHIP, standalone Prolog applications, and even applications not written in Prolog. It has certainly proven very useful for documenting library modules. However, we have also found it quite useful for generating "internals" and also user manuals of applications and project reports. Because the system can not only generate manuals in many formats, but also maintain documentation and software distribution sites, we have found ourselves using it for documenting and building such sites for a number of applications which, as mentioned above, were not even written in Prolog.

We have found that, with a bit of practice, one can write assertions and comments that at the same time document the program code, document the external use of the library, and greatly improve the debugging and maintenance cycles. One of the fundamental practical advantages observed when using lpdoc to document programs is that it is much easier to maintain a true correspondence between the program and its documentation, and to identify precisely to what version of the program a given printed manual corresponds. Furthermore, another fundamental advantage comes from the fact that the assertions are designed to be checkable in part, either statically or dynamically $[10,8]$, so that the documentation also achieves a certain degree of certification. While in the Ciao system writing assertions is optional (in contrast to, e.g., Mercury [15]), the fact that they will generate a good part of the manual encourages programmers to write them, and this in turn helps developing programs faster, because more errors are detected early on.

lpdoc is publicly available. ${ }^{10}$ The system is currently undergoing further development in several directions, such as, for example, reducing the need for auxiliary applications (so that it is portable to more platforms) or improving the emacs-based interactive environment. As mentioned previously, with a simple compatibility library it is relatively easy to make traditional (constraint) logic programming systems (in which new declarations can be defined) accept programs adorned with Ciao-style assertions and comments, so that they are ignored during compilation but lpdoc (and the Ciao preprocessor!) can be used on them. As mentioned above, we have done this for SICStus and CHIP. It should not be too difficult to modify the front end for other type/assertion languages, such as those used in Mercury [15] and HAL [5] (this is under study at least in the case of HAL), or even non LP-based languages (which would, however, need a specific front-end).

${ }_{10}$ See http://www.clip.dia.fi.upm.es/Software . 


\section{References}

1. F. Bueno, D. Cabeza, M. Carro, M. Hermenegildo, P. López-García, and G. Puebla. The Ciao Prolog System. Reference Manual. TR CLIP3/97.1, School of Computer Science, Technical University of Madrid (UPM), August 1997.

2. D. Cabeza and M. Hermenegildo. A New Module System for Prolog. In International Conference on Computational Logic, CL2000, LNCS. Springer-Verlag, July 2000. To appear.

3. D. Cabeza and M. Hermenegildo. The Ciao Modular, Standalone Compiler and Its Generic Program Processing Library. In Special Issue on Parallelism and Implementation of (C)LP Systems. To appear, Electronic Notes in Theoretical Computer Science. Elsevier - North Holland, 2000.

4. D. Cordes and M. Brown. The Literate Programming Paradigm. IEEE Computer Magazine, June 1991.

5. B. Demoen, M. Garcia de la Banda, W. Harvey, K. Marriott, and P. Stuckey. Herbrand Constraint Solving in HAL. In Int. Conf. on Logic Programming. MIT Press, Cambridge, MA, U.S.A., November 1999.

6. P. Deransart, A. Ed-Dbali, and L. Cervoni. Prolog: The Standard. Springer-Verlag, 1996.

7. Lisa Friendly. The Design of Distributed Hyperlink Program Documentation. In Int'l. WS on Hypermedia Design, Workshops in Computing. Springer, June 1996. Available from http://java.sun.com/docs/javadoc-paper.html.

8. M. Hermenegildo, F. Bueno, G. Puebla, and P. López-García. Program Analysis, Debugging and Optimization Using the Ciao System Preprocessor. In 1999 International Conference on Logic Programming, pages 52-66, Cambridge, MA, November 1999. MIT Press.

9. M. Hermenegildo and The CLIP Group. An Automatic Documentation Generator for (C)LP - Reference Manual. The Ciao System Documentation Series-TR CLIP5/97.3, Facultad de Informática, UPM, August 1997.

10. M. Hermenegildo, G. Puebla, and F. Bueno. Using Global Analysis, Partial Specifications, and an Extensible Assertion Language for Program Validation and Debugging. In K. R. Apt, V. Marek, M. Truszczynski, and D. S. Warren, editors, The Logic Programming Paradigm: a 25-Year Perspective, pages 161-192. SpringerVerlag, July 1999.

11. D. Knuth. Literate programming. Computer Journal, 27:97-111, 1984.

12. G. Puebla, F. Bueno, and M. Hermenegildo. An Assertion Language for Debugging of Constraint Logic Programs. In ILPS'97 WS on Tools and Environments for (C)LP, October 1997. ftp://clip.dia.fi.upm.es/pub/papers/assert/lang_tr_discipldeliv.ps.gz

13. G. Puebla, F. Bueno, and M. Hermenegildo. An Assertion Language for Debugging of Constraint Logic Programs. In P. Deransart, M. Hermenegildo, and J. Maluszynski, editors, Analysis and Visualization Tools for Constraint Programming, LNCS. Springer-Verlag, 2000. To appear.

14. G. Puebla and M. Hermenegildo. Some Issues in Analysis and Specialization of Modular Ciao-Prolog Programs. In Special Issue on Optimization and Implementation of Declarative Programming Languages, volume 30 of Electronic Notes in Theoretical Computer Science. Elsevier - North Holland, March 2000.

15. Z. Somogyi, F. Henderson, and T. Conway. The execution algorithm of Mercury: an efficient purely declarative logic programming language. $J L P, 29(1-3)$, October 1996.

Note: most CLIP group publications are available from http://www.clip.dia.fi.upm.es 


\section{A An example: source}

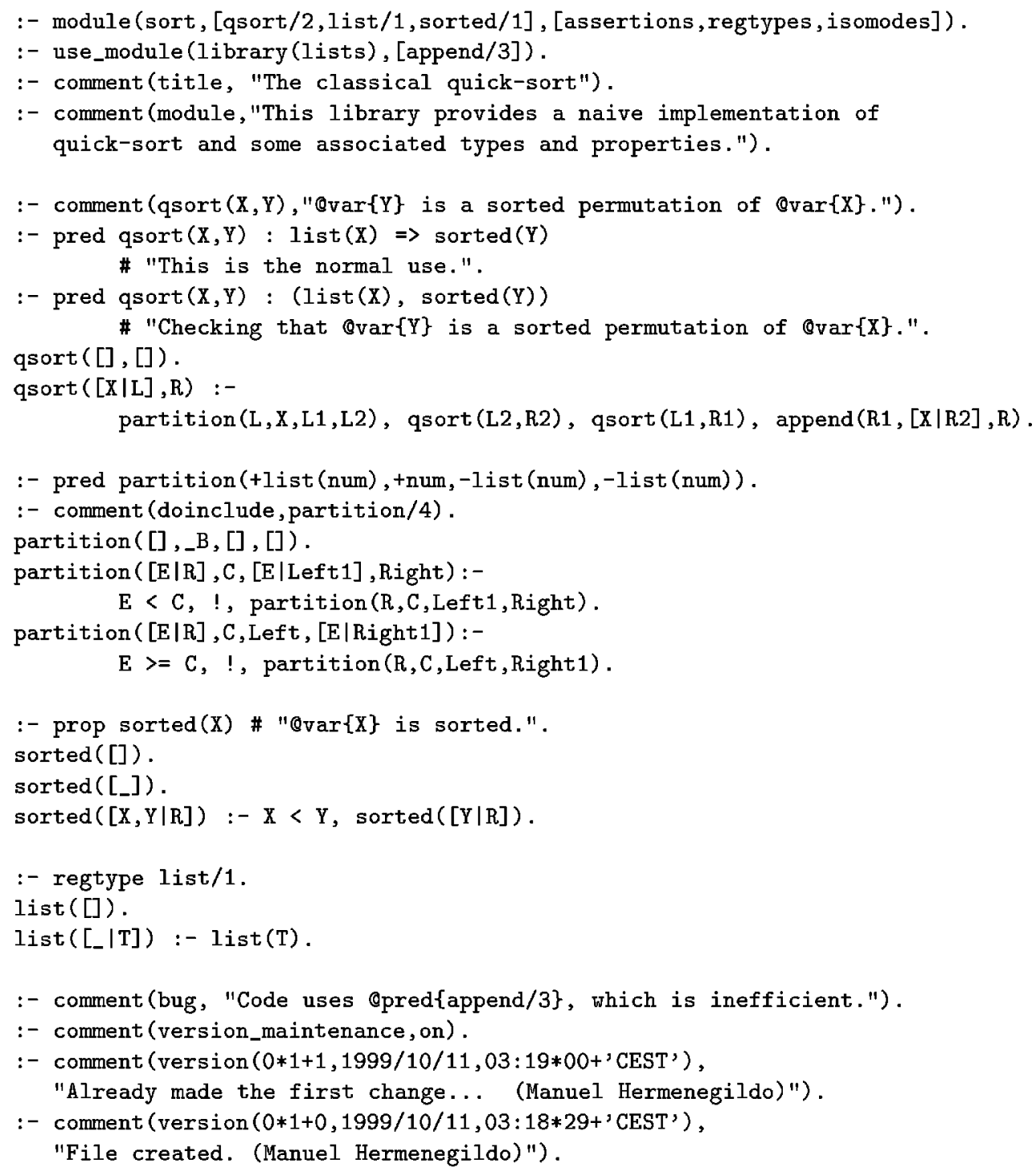

\section{B The classical quick-sort}

Version: 0.1\#1 (1999/10/11, 3:19:0 CEST)

This library provides a naive implementation of quick-sort and some associated types and properties.

\section{B.1 Usage and interface (sort)}

\footnotetext{
- Library usage: :- use_module (library (sort)).
} 
- Exports:

- Predicates:

qsort $/ 2$.

- Properties:

sorted/1.

- Regular Types:

list/1.

- Other modules used:

- System library modules: lists.

\section{B.2 Documentation on exports (sort)}

qsort/2:

PREDICATE

qsort $(X, Y): Y$ is a sorted permutation of $X$.

Usage 1: $q \operatorname{sort}(X, Y)$

- Description: This is the normal use.

- Should hold at call time: list(X).

- Should hold upon exit: $\mathrm{Y}$ is sorted (sorted/1).

Usage 2: $q \operatorname{sort}(\mathrm{X}, \mathrm{Y})$

- Description: Checking that $\mathrm{Y}$ is a sorted permutation of $\mathrm{X}$.

- Should hold at call time: list(X) (list/1), Y is sorted (sorted/1).

list / 1:

REGTYPE

A regular type, defined as follows:

list( []$)$.

list $\left(\left[\_1 \mid T\right]\right)$ :-

list $(\mathrm{T})$.

sorted/1:

PROPERTY

Usage: sorted (X)

- Description: $\mathrm{X}$ is sorted.

\section{B.3 Documentation on internals (sort)}

\section{partition/4:}

PREDICATE

\section{Usage:}

- Should hold at call time: Arg1 is a list of nums (list/2), Arg2 is a number (list/2), Arg3 is a free variable (var/1), Arg4 is a free variable (var/1).

- Should hold upon exit: Arg3 is is a list of nums (list/2), Arg4 is is a list of nums (list/2).

B.4 Known bugs and planned improvements (sort)

- Code uses append/3, which is inefficient.

B.5 Version/Change Log (sort)

- Version 0.1\#1 (1999/10/11, 3:19:0 CEST)

Already made the first change... (Manuel Hermenegildo)

- Version 0.1 (1999/10/11, 3:18:29 CEST)

File created. (Manuel Hermenegildo) 